\title{
Transport properties for a Luttinger liquid wire with Rashba spin-orbit coupling and Zeeman splitting
}

\author{
Fang Cheng ${ }^{1,2}$ and Guanghui Zhou ${ }^{1,2,3 *}$ \\ ${ }^{1}$ CCAST (World Laboratory), PO Box 8730, Beijing 100080, China \\ ${ }^{2}$ Department of Physics, Hunan Normal University, Changsha 410081, China ${ }^{\dagger}$ and \\ ${ }^{3}$ International Center for Materials Physics, Chinese Academy of Sciences, Shenyang 110015, China
}

\begin{abstract}
We study the transport properties for a Luttinger-liquid (LL) quantum wire in the presence of both Rashba spin-orbit coupling (SOC) and a weak external in-plane magnetic field. The bosonized Hamiltonian of the system with an externally applied longitudinal electric field is established. And then the equations of motion for the bosonic phase fields are solved in the Fourier space, with which the both charge and spin conductivities for the system are calculated analytically based on the linear response theory. Generally, the ac conductivity is an oscillation function of the strengths of electron-electron interaction, Rashba SOC and magnetic field, as well as the driving frequency and the measurement position in the wire. Through analysis with some examples it is demonstrated that the modification on the conductivity due to electron-electron interactions is more remarkable than that due to SOC, while the effects of SOC and Zeeman splitting on the conductivity are very similar. The spin-polarized conductivities for the system in the absence of Zeeman effect or SOC are also discussed, respectively. The ratio of the spin-polarized conductivities $\sigma_{\uparrow} / \sigma_{\downarrow}$ is dependent of the electron-electron interactions for the system without SOC, while it is independent of the electron-electron interactions for the system without Zeeman splitting.
\end{abstract}

PACS numbers: 73.23.-b, 71.10.Pm, 71.70.Ej, 73.63.Nm

\section{INTRODUCTION}

The physics of one dimensional (1D) systems of strongly correlated particles has become a very interesting subject because of the simplicity of the models and the attainment of the truly $1 \mathrm{D}$ systems due to breakthrough in material technology. From the theoretical point of view, Luttinger-liquid (LL) model is appropriate to describe the transport properties of $1 \mathrm{D}$ systems with electron-electron interactions. ${ }^{1}$ The LL model is of fundamental importance because it is one of very few strongly correlated "non Fermi liquid" systems that can be analyzed in any detail. The model does not attempt a complete description of electrons in a 1D metal but rather confines to the vicinity of the Fermi surface. One of the key features of the LL model is the spin-charge separation: the low-energy excitations are not quasiparticles with charge $e$ and spin $\hbar / 2$ together but collective modes of spin and charge excitation separately. Therefore, quantum transport in LL systems has attracted a great deal of interest since the experimental realization of the narrow quantum wire formed in semiconductor heterostructures $^{2}$ and the carbon nanotube ${ }^{3}$ as well as the edge states of the fractional Quantum Hall liquid. ${ }^{4}$ We will use the first one as our physical subject in this work.

Spintronics is a multidisciplinary field whose central theme is the active manipulation of spin degrees of freedom in solid state systems. It is believed to be a promising candidate for the future information technology. ${ }^{5}$

${ }^{\dagger}$ Mailing address
There are two physical mechanisms which can be used to influence the dynamics of the electron spin in normal conductors, i.e., spin-orbit coupling (SOC) and Zeeman splitting. In layered semiconductors devices, the two predominant types of SOC are Dresselhaus $\mathrm{SOC}^{6}$ and Rashba SOC. ${ }^{7}$ The former arises from the breaking of inversion symmetry by the inherent asymmetry of the atomic arrangement in the structure and is not very amenable to external manipulation. The latter, on the other hand, arises from band bending at the interfaces between semiconductor layers and/or any external electric fields applied to the device. Unlike Dresselhaus SOC, the strength of the Rashba SOC can be partially controlled by application of an external electric field perpendicular to the two-dimensional electron gas (2DEG) plane. ${ }^{8}$ And in many of the proposed spintronics device structures the spin manipulation relies on the Rashba SOC, and as such, only the Rashba SOC will be considered in our work.

In recent few years, there have been tremendous published research works of the SOC effects on the III-V type and II-VI type nonmagnetic semiconductor heterostructures for the purpose of spintronics devices. But there have been only few works ${ }^{9-15}$ concerning electronelectron interactions in these spintronic systems. The early theoretical studies ${ }^{9}$ demonstrated that the influence of the Zeeman splitting for a LL quantum wire is the breaking of spin-charge separation, where the ratio of the spin-up and spin-down conductivities in a dirty system diverges at low temperatures due to the electron correlation and results in a spin-polarized current. Further studies ${ }^{10-12}$ also have shown that the effect of Rashba SOC for the LL wire is that the spin degeneracy is lifted for $k \neq 0$ and each branch loses its vertical symmetry axis, i.e., different directions of motion 
have different Fermi velocities. Moreover, Coulomb corrections to extrinsic spin Hall-effect of a 2DEG has also been studied recently. ${ }^{13}$ In methodology, a bosonization theory including a Rashba $\mathrm{SOC}^{10}$ or a Zeeman splitting ${ }^{9}$ has been constructed. A further question arising naturally is what will happen if both the SOC and Zeeman splitting are considered simultaneously. This motivation has led to the studies ${ }^{14,15}$ on the combined presence of a Rashba SOC and a Zeeman effect in an interacting quantum wire. In these works, the study on a Coulomb longranged electron interaction quantum wire ${ }^{15}$ in the combined presence of a Rashba SOC and a Zeeman splitting using the perturbative renormalization group treatment has indicated the generation of a spin pseudogap and the propagation of a well-defined spin-oriented current, and on a LL quantum wire ${ }^{14}$ by bosonization technique has demonstrated that the tunneling current may deviate from a simple power law which is that in an ordinary LL wire.

On the other hand, however, the pure LL quantum wire without SOC and Zeeman splitting has been extensively investigated in last decade. The ac response of $1 \mathrm{D}$ interacting system has been studied ${ }^{16-18}$ previously in the framework of the LL model with or without impurity. Moreover, it is known that the dc limit of conductance through a clean LL quantum wire is not renormalized by the electron-electron interactions when the reservoirs (leads) are properly taken into account because a wellknown phenomenon of Andreev-type reflection occurring at the contact between a LL quantum wire and a noninteracting reservoir. ${ }^{19}$

In this work, we study the ac dynamical transport properties for a homogenous interacting quantum wire in the presence of both internal Rashba SOC and external magnetic field simultaneously. When a longitudinal time-varying electric field is applied to the wire, in the LL regime we use the bosonization technique and solve the equation of motion for the system in the Fourier space. It is found that in this case the spin and charge degrees of freedom are completely coupled and can be characterized by four new different velocities. Within the linear response theory, the dynamical ac $(\omega \neq 0)$ conductivity of the system is generally an oscillation function of the strengths of electron-electron interaction, Rashba SOC and Zeeman interaction as well as the driving frequency and the measurement position in the wire. However, the modification of $g$ on the conductivity is more remarkable than the modification on the conductivity due to electron-electron interactions is more remarkable than that due to SOC, while the effects of SOC and Zeeman splitting on the conductivity are very similar. The spinpolarized conductivities for the system in the absence of Zeeman effect or SOC are also discussed, respectively. The ratio of the spin-polarized conductivities $\sigma_{\uparrow} / \sigma_{\downarrow}$ is dependent of the electron-electron interactions for the system without SOC, and the ratio becomes more different when the electron-electron interactions are stronger, while it is independent of the electron-electron interac- tions for the system without Zeeman splitting. To the best of our knowledge, some of these phenomena have not been reported previously for the LL quantum wire system.

The rest of the paper is organized as follows. In Sec. II, we formulate the model Hamiltonian in the bosonization form for an interacting quantum wire simultaneously with an external longitudinal electric field applied and both Rashba SOC and Zeeman splitting, and solve the equation of motion for the bosonic phase fields in the Fourier space. Within the linear response theory, the conductivity of the system is analytically calculated in Sec. III, and the detailed results for the two limited cases without either Zeeman splitting or Rashba SOC are demonstrated in two subsections, respectively. Some examples and the discussion of the results are demonstrated in Sec. IV. Finally, Sec. V concludes the paper.

\section{THE HAMILTONIAN AND BOSONIZATION}

Consider the system consisting of an interacting 1D quantum wire with an applied longitudinal electric field realized by an externally applied electromagnetic radiation in the experience. In the $1 \mathrm{D}$ quantum wire the electron is subjected to a Rashba SOC. Here we have taken the symmetric center of the quantum wire as the origin and the growth direction of the heterostructure to be the $z$ axis in our spatial coordinate system. The electron transport ballistically in the quantum wire along the longitudinal $x$ direction. A magnetic field $B$ perpendicular to the quantum wire is applied along $y$ axis.

For a weak magnetic field, its coupling to the electron orbital can be neglected ${ }^{14}$ if the low-lying excitation is considered, so we only keep the Zeeman Hamiltonian term with respect to the magnetic field. Assuming $\delta v_{R} \sim \delta v_{B} \ll v_{F}$, the linearized noninteracting electron Hamiltonian of the quantum wire with both Rashba SOC and Zeeman splitting is given by ${ }^{9,10,14}$

$$
H_{0}=-i \hbar \int \sum_{\gamma, s} v_{\gamma}^{s} \psi_{\gamma s}^{+} \partial_{x} \psi_{\gamma s} d x
$$

The operators $\psi_{\gamma s}(\gamma=L, R ; s=\downarrow, \uparrow)$ annihilate spindown $(\downarrow)$ and spin-up ( $\uparrow)$ electrons near the left (L) and right $(R)$ Fermi points. In what follows, the indices $\gamma$ and $s$ take the values $-1(1)$ for $L(R)$ and $\downarrow(\uparrow)$, respectively. And $v_{\gamma}^{s}=\gamma v_{F}-\frac{1}{2} s \delta v_{R}+\frac{1}{2} \gamma s \delta v_{B}$ are four different sound velocities. Here $v_{F}$ is the bare Fermi velocity of noninteracting right and left movers, $\delta v_{R}=2 \alpha / \hbar(\alpha$ is the strength of Rashba SOC) and $\delta v_{B}=g^{\prime} \mu_{B} B / k_{F}(B$ is the magnitude of magnetic field, $g^{\prime}$ is the Lande factor, and $\mu_{B}$ is the Bohr magneton, respectively). Eq. (11) shows clearly that the Rashba term splits horizontally the bands and makes the electron Fermi velocities become different for different directions of motion, while the Zeeman term splits vertically the bands and makes the electron Fermi velocities become different for different 
directions of spin. Using the bosonization technique ${ }^{20}$ in terms of

$$
\psi_{\gamma s}^{+} \partial_{x} \psi_{\gamma s}=i \gamma\left(\frac{\gamma \partial_{x} \vartheta_{s}-\frac{\Pi_{s}}{\hbar}}{2}\right)^{2},
$$

we can derive Hamiltonian (1) as

$$
\begin{aligned}
H_{0} & =\frac{\hbar v_{F}}{2} \int d x\left[\left(\partial_{x} \vartheta_{\uparrow}\right)^{2}+\left(\frac{\Pi_{\uparrow}}{\hbar}\right)^{2}+\left(\partial_{x} \vartheta_{\downarrow}\right)^{2}+\left(\frac{\Pi_{\downarrow}}{\hbar}\right)^{2}\right] \\
& -\frac{\hbar}{2} \delta v_{B} \int d x\left[\left(\partial_{x} \vartheta_{\downarrow}\right)^{2}+\left(\frac{\Pi_{\downarrow}}{\hbar}\right)^{2}-\left(\partial_{x} \vartheta_{\uparrow}\right)^{2}-\left(\frac{\Pi_{\uparrow}}{\hbar}\right)^{2}\right] \\
& +\frac{\delta v_{R}}{2} \int d x\left[\Pi_{\uparrow}\left(\partial_{x} \vartheta_{\uparrow}\right)-\Pi_{\downarrow}\left(\partial_{x} \vartheta_{\downarrow}\right)\right],
\end{aligned}
$$

where $\vartheta_{\uparrow / \downarrow}$ is the phase field for spin-up/down electrons and $\Pi_{\uparrow / \downarrow}$ is the corresponding conjugate momentum. With the transformation

$\vartheta_{\rho}=\frac{\vartheta_{\uparrow}+\vartheta_{\downarrow}}{\sqrt{2}}, \vartheta_{\sigma}=\frac{\vartheta_{\uparrow}-\vartheta_{\downarrow}}{\sqrt{2}}, \Pi_{\rho}=\frac{\Pi_{\uparrow}+\Pi_{\downarrow}}{\sqrt{2}}, \Pi_{\sigma}=\frac{\Pi_{\uparrow}-\Pi_{\downarrow}}{\sqrt{2}}$,

we can reduce Eq. (3) into

$$
\begin{aligned}
H_{0} & =\frac{\hbar v_{F}}{2} \int d x\left[\left(\partial_{x} \vartheta_{\rho}\right)^{2}+\left(\frac{\Pi_{\rho}}{\hbar}\right)^{2}+\left(\partial_{x} \vartheta_{\sigma}\right)^{2}+\left(\frac{\Pi_{\sigma}}{\hbar}\right)^{2}\right] \\
& +\frac{\hbar}{2} \delta v_{B} \int d x\left[\left(\partial_{x} \vartheta_{\sigma}\right)\left(\partial_{x} \vartheta_{\rho}\right)+\frac{1}{\hbar^{2}} \Pi_{\rho} \Pi_{\sigma}\right] \\
& +\frac{\delta v_{R}}{2} \int d x\left[\Pi_{\sigma}\left(\partial_{x} \vartheta_{\rho}\right)+\Pi_{\rho}\left(\partial_{x} \vartheta_{\sigma}\right)\right],
\end{aligned}
$$

where $\vartheta_{\rho}$ and $\vartheta_{\sigma}$ can be considered as the phase field corresponding to the charge degree and the spin degree of freedom, respectively, and $\Pi_{\rho}$ and $\Pi_{\sigma}$ are the corresponding conjugate momentums.

Next, the short-ranged electron-electron interactions in the wire give a term to the Hamiltonian

$$
H_{i n t}=\frac{V(q=0)}{2 \pi} \int d x\left(\partial_{x} \vartheta_{\rho}\right)^{2},
$$

where $V(q=0)$ is the electron-electron interaction potential. In this Hamiltonian we have neglected the Umklapp scattering, which is not relevant in the quantum wires formed in semiconductor heterostructure.

Finally, we consider the Hamiltonian term of a longitudinal electric field applied to the quantum wire. We use the method of describing the application of an external bias voltage. ${ }^{19}$ With the electron charge $-e$, the coupling to an external time-dependent potential $U_{R}(t)$ yields a term in the Hamiltonian as ${ }^{17}$

$$
H_{a c}=-e \int d x \rho(x) U_{R}(x, t)=\sqrt{\frac{2}{\pi}} e \int d x \partial_{x} U_{R}(x, t) \vartheta_{\rho},
$$

where $U_{R}(x, t)$ is the chemical potential of the right moving electrons, $\rho(x, t)=\sqrt{\frac{2}{\pi}} \partial_{x} \vartheta_{\rho}(x, t)$ is the charge density in bosonization presentation. By virtue of the relation $\partial_{x} U_{R}(x, t)=-E(x, t)$, Eq. (7) can be expressed as

$$
H_{a c}=-\sqrt{\frac{2}{\pi}} e \int d x E(x, t) \vartheta_{\rho}(x, t),
$$

where $E(x, t)$ is the externally applied electric field.

Combining Eqs. (5), (6) and (8), we finally obtain the total bosonized Hamiltonian for the system

$$
\begin{aligned}
H & =\frac{\hbar}{2} \int d x\left[\frac{v_{\rho}}{g}\left(\partial_{x} \vartheta_{\rho}\right)^{2}+v_{F}\left(\frac{\Pi_{\rho}}{\hbar}\right)^{2}\right] \\
& +\frac{\hbar}{2} \int d x\left[v_{\sigma}\left(\partial_{x} \vartheta_{\sigma}\right)^{2}+v_{\sigma}\left(\frac{\Pi_{\sigma}}{\hbar}\right)^{2}\right] \\
& \left.+\frac{\hbar}{2} \delta v_{B} \int d x\left[\left(\partial_{x} \vartheta_{\sigma}\right)\left(\partial_{x} \vartheta_{\rho}\right)+\frac{1}{\hbar^{2}} \Pi_{\rho} \Pi_{\sigma}\right)\right] \\
& +\frac{\hbar}{2} \delta v_{R} \int d x\left[\left(\frac{\Pi_{\sigma}}{\hbar}\right)\left(\partial_{x} \vartheta_{\rho}\right)+\left(\frac{\Pi_{\rho}}{\hbar}\right)\left(\partial_{x} \vartheta_{\sigma}\right)\right] \\
& -\sqrt{\frac{2}{\pi}} e \int d x E(x, t) \vartheta_{\rho}(x, t),
\end{aligned}
$$

where $v_{\rho, \sigma}$ are the propagation velocities of the charge and spin collective modes of the decoupled model $\left(\delta v_{B}=\right.$ $\delta v_{R}=0$, or $B=\alpha=0$ ) and the parameter $g$ is the strength of the electron-electron interactions, which is defined as $1 / g^{2}=1+V(q=0) / \hbar \pi v_{F}$ with $v_{F}$ is the non-interacting Fermion velocity of the system, noninteracting Fermions corresponds to $g=1$ and repulsive interaction corresponds to $g<1$. The velocities $v_{\rho, \sigma}$ have been obtained as function of $g$ and $v_{F}$ in Ref.[20] as $v_{\rho}=v_{F} / g$ and $v_{\sigma}=v_{F}$ for the decoupled model.

Further, the action functional of the coupled system can be written in terms of the phase fields $\vartheta_{\rho}(x, t)$ and $\vartheta_{\sigma}(x, t)$ as

$$
\begin{aligned}
S & =\frac{\hbar}{2} \int d t \int d x\left[\frac{1}{g v_{\rho}}\left(\partial_{t} \vartheta_{\rho}\right)^{2}-\frac{v_{\rho}}{g}\left(\partial_{x} \vartheta_{\rho}\right)^{2}\right] \\
& +\frac{\hbar}{2} \int d t \int d x\left[\frac{1}{v_{\sigma}}\left(\partial_{t} \vartheta_{\sigma}\right)^{2}-v_{\sigma}\left(\partial_{x} \vartheta_{\sigma}\right)^{2}\right] \\
& -\frac{\hbar}{2} \frac{\delta v_{R}}{v_{F}} \int d t \int d x\left[\left(\partial_{t} \vartheta_{\sigma}\right)\left(\partial_{x} \vartheta_{\rho}\right)+\left(\partial_{t} \vartheta_{\rho}\right)\left(\partial_{x} \vartheta_{\sigma}\right)\right] \\
& -\frac{\hbar}{2} \delta v_{B} \int d x\left[\left(\partial_{x} \vartheta_{\sigma}\right)\left(\partial_{x} \vartheta_{\rho}\right)+\frac{1}{\left(v_{F}\right)^{2}}\left(\partial_{t} \vartheta_{\rho}\right)\left(\partial_{t} \vartheta_{\sigma}\right)\right] \\
& +\sqrt{\frac{2}{\pi}} e \int d x E(x, t) \vartheta_{\rho}(x, t) .
\end{aligned}
$$

Note that in our system the time derivative of the field is not anymore proportional to the conjugate canonical momentum, but is a linear combination of the canonical momentum (including charge canonical momenta and spin canonical momenta) and the gradient of the field. However, after omitting the second power of the shifts from the spin-obit or the Zeeman term and the product between them, we find that the extra charge or spin canonical momentum and the gradient of the field produce the same terms in the first line of Eq. (9) and the first term $\left(\Pi_{\rho} \partial_{t} \vartheta_{\rho}+\Pi_{\sigma} \partial_{t} \vartheta_{\sigma}\right)$ of the Lagrangian $L=\int d x\left(\Pi_{\rho} \partial_{t} \vartheta_{\rho}+\Pi_{\sigma} \partial_{t} \vartheta_{\sigma}\right)-H$, which finally make 
that the extra charge or spin canonical momentum and the gradient of the field have not effect on the action functional of the coupled system. Therefore, by minimizing action (10) we obtain its associated equations of motion for the phase fields

$$
\begin{array}{r}
\frac{\hbar}{g v_{\rho}} \partial_{t}^{2} \vartheta_{\rho}-\frac{\hbar v_{\rho}}{g} \partial_{x}^{2} \vartheta_{\rho}-\hbar \frac{\delta v_{R}}{v_{F}} \partial_{t} \partial_{x} \vartheta_{\sigma}-\frac{\hbar}{2} \delta v_{B} \partial_{x}^{2} \vartheta_{\sigma}-\frac{\hbar}{2} \frac{\delta v_{B}}{v_{F}^{2}} \partial_{t}^{2} \vartheta_{\sigma}+\sqrt{\frac{2}{\pi}} e E(x, t)=0 \\
\frac{\hbar}{v_{\sigma}} \partial_{t}^{2} \vartheta_{\sigma}-\hbar v_{\sigma} \partial_{x}^{2} \vartheta_{\sigma}-\hbar \frac{\delta v_{R}}{v_{F}} \partial_{t} \partial_{x} \vartheta_{\rho}-\frac{\hbar}{2} \delta v_{B} \partial_{x}^{2} \vartheta_{\rho}-\frac{\hbar}{2} \frac{\delta v_{B}}{v_{F}^{2}} \partial_{t}^{2} \vartheta_{\rho}=0 .
\end{array}
$$

Applying the Fourier transformation

$$
\vartheta(x, t)=\frac{1}{(2 \pi)^{2}} \int d q \int d \omega \vartheta(q, \omega) e^{-i q x+i \omega t}
$$

to Eqs. (11) and (12), we have the solution for the phase fields

$$
\begin{gathered}
\vartheta_{\rho}(q, \omega)=\sqrt{\frac{2}{\pi} \frac{e v_{F}}{\hbar}} \frac{E(q, \omega)}{\left(\omega^{2}-v_{\rho}^{2} q^{2}\right)-\frac{\left(\delta v_{R} q \omega-\frac{1}{2} \delta v_{B} v_{F} q^{2}-\frac{1}{2} \frac{\delta v_{B}}{v_{F}} \omega^{2}\right)^{2}}{\omega^{2}-v_{\sigma}^{2} q^{2}}} \\
\vartheta_{\sigma}(q, \omega)=-\sqrt{\frac{2}{\pi} \frac{e v_{F}}{\hbar}} \frac{\left(\delta v_{R} q \omega-\frac{1}{2} \delta v_{B} v_{F} q^{2}-\frac{1}{2} \frac{\delta v_{B}}{v_{F}} \omega^{2}\right) E(q, \omega)}{\left(\omega^{2}-v_{\rho}^{2} q^{2}\right)\left(\omega^{2}-v_{\sigma}^{2} q^{2}\right)-\left(\delta v_{R} q \omega-\frac{1}{2} \delta v_{B} v_{F} q^{2}-\frac{1}{2} \frac{\delta v_{B}}{v_{F}} \omega^{2}\right)^{2}} .
\end{gathered}
$$

\section{CONDUCTIVITY OF THE SYSTEM}

The current operator can be defined by using the $1 \mathrm{D}$ continuity equation $\partial_{x} j_{\rho}(x, t)=e \partial_{t} \rho(x, t)$. Then we have the charge current

$$
j_{\rho}(x, t)=\sqrt{\frac{2}{\pi}} e \partial_{t} \vartheta_{\rho}(x, t) .
$$

Therefore, using solution (14) for $\vartheta_{\rho}(q, \omega)$, we obtain the explicit expression for the charge current operator

$$
j_{\rho}(q, \omega)=\frac{i e^{2} v_{F}}{\hbar \pi} \frac{2 \omega\left(\omega^{2}-v_{\sigma}^{2} q^{2}\right) E(q, \omega)}{\left(\omega^{2}-v_{\rho}^{2} q^{2}\right)\left(\omega^{2}-v_{\sigma}^{2} q^{2}\right)-\left(\delta v_{R} q \omega-\frac{1}{2} \delta v_{B} v_{F} q^{2}-\frac{1}{2} \frac{\delta v_{B}}{v_{F}} \omega^{2}\right)^{2}},
$$

And the charge current operator is written further as

$$
j_{\rho}(q, \omega)=\frac{i e^{2} v_{F}}{\hbar \pi} \frac{i E(q, \omega)}{1-\frac{\delta v_{B}^{2}}{4 v_{F}^{2}}} \frac{2 \omega\left(\omega^{2}-v_{\sigma}^{2} q^{2}\right)}{\left(\omega+u_{1} q\right)\left(\omega+u_{2} q\right)\left(\omega+u_{3} q\right)\left(\omega+u_{4} q\right)},
$$

where $u_{1,2,3,4}$ are the velocities of four independent branches of the chiral excitations, and they are all related to $g, \delta v_{R}$ and $\delta v_{B} \cdot{ }^{14}$ Since linear response is exact for an ideal LL, the external electric field has to be used 
for the conductivity calculation, ${ }^{18}$ i.e.,

$$
j_{\rho}(q, \omega)=\sigma(q, \omega) E(q, \omega) .
$$

Therefore, combining Eq. (17) with Eq. (19), we obtain the nonlocal charge conductivity

$$
\sigma_{\rho}(q, \omega)=\frac{i e^{2} v_{F}}{\hbar \pi} \frac{2 \omega\left(\omega^{2}-v_{\sigma}^{2} q^{2}\right)}{\left(\omega^{2}-v_{\rho}^{2} q^{2}\right)\left(\omega^{2}-v_{\sigma}^{2} q^{2}\right)-\left(\delta v_{R} q \omega-\frac{1}{2} \delta v_{B} v_{F} q^{2}-\frac{1}{2} \frac{\delta v_{B}}{v_{F}} \omega^{2}\right)^{2}}
$$

On the other hand, the bosonic phase field $\vartheta_{\sigma}$ is related to the spin current operator through

$$
j_{\sigma}=\sqrt{\frac{2}{\pi}} e \partial_{t} \vartheta_{\sigma}
$$

Combing Eqs. (15) and (21), the spin current operator can be expressed as

$$
j_{\sigma}(q, \omega)=\frac{i e^{2} v_{F}}{\hbar \pi} \frac{2 \omega\left(\delta v_{R} q \omega-\frac{1}{2} \delta v_{B} v_{F} q^{2}-\frac{1}{2} \frac{\delta v_{B}}{v_{F}} \omega^{2}\right) E(q, \omega)}{\left(\omega^{2}-v_{\rho}^{2} q^{2}\right)\left(\omega^{2}-v_{\sigma}^{2} q^{2}\right)-\left(\delta v_{R} q \omega-\frac{1}{2} \delta v_{B} v_{F} q^{2}-\frac{1}{2} \frac{\delta v_{B}}{v_{F}} \omega^{2}\right)^{2}}
$$

Therefore, using the linear response relation (19), we can also obtain the spin conductivity

$$
\sigma_{\sigma}(q, \omega)=\frac{i e^{2} v_{F}}{\hbar \pi} \frac{2 \omega\left(\delta v_{R} q \omega-\frac{1}{2} \delta v_{B} v_{F} q^{2}-\frac{1}{2} \frac{\delta v_{B}}{v_{F}} \omega^{2}\right)}{\left(\omega^{2}-v_{\rho}^{2} q^{2}\right)\left(\omega^{2}-v_{\sigma}^{2} q^{2}\right)-\left(\delta v_{R} q \omega-\frac{1}{2} \delta v_{B} v_{F} q^{2}-\frac{1}{2} \frac{\delta v_{B}}{v_{F}} \omega^{2}\right)^{2}}
$$

which is also a function of $g, \alpha, B, \omega$ and $q$.

Next, for understanding the transport property of the system in more detail, we go further for the two limited cases of $B=0$ or $\alpha=0$, respectively, in the following subsections.

\section{A. The conductivity with Rashba SOC}

Consider a LL quantum wire submitted to Rashba SOC without a Zeeman splitting, i.e., in the absence of external magnetic field $\left(B=0\right.$ or $\left.\delta v_{B}=0\right)$. In this case the expression of the current operator Eq. (17) is reduced to

$$
j_{\rho}(q, \omega)=\frac{i e^{2} v_{F}}{\hbar \pi} \frac{2 \omega\left(\omega^{2}-v_{\sigma}^{2} q^{2}\right) E(q, \omega)}{\left(\omega^{2}-u_{1}^{2} q^{2}\right)\left(\omega^{2}-u_{2}^{2} q^{2}\right)},
$$

where

$$
u_{1,2}^{2}=\frac{\delta v_{R}^{2}+v_{\rho}^{2}+v_{\sigma}^{2}}{2} \pm \frac{\sqrt{\left(\delta v_{R}^{2}+v_{\rho}^{2}+v_{\sigma}^{2}\right)^{2}-4 v_{\rho}^{2} v_{\sigma}^{2}}}{2}
$$

are the propagation velocities of coupled collective modes in which the subscript $1 / 2$ corresponds to +/-. Furthermore, in the absence of SOC $\left(\alpha=0\right.$ or $\left.\delta v_{R}=0\right)$, we simply have $u_{1,2}=v_{\rho, \sigma}$ which correspond to the velocities for the special case of spin-charge separation in a LL quantum wire. ${ }^{16}$ Moreover, Eq.(24) can be rewritten as

$$
\begin{aligned}
j_{\rho}(q, \omega) & =\frac{i e^{2} v_{F} E(q, \omega)}{\hbar \pi}\left[\frac{u_{1}^{2}-v_{\sigma}^{2}}{u_{1}^{2}-u_{2}^{2}}\left(\frac{1}{\omega+u_{1} q}+\frac{1}{\omega-u_{1} q}\right)\right. \\
& \left.-\frac{u_{2}^{2}-v_{\sigma}^{2}}{u_{1}^{2}-u_{2}^{2}}\left(\frac{1}{\omega+u_{2} q}+\frac{1}{\omega-u_{2} q}\right)\right] .
\end{aligned}
$$

Therefore, combining Eq. (19) with Eq. (26), we ob- 
tain the nonlocal charge conductivity

$$
\begin{aligned}
\sigma_{\rho}(q, \omega) & =\frac{i e^{2} v_{F}}{\hbar \pi}\left[\frac{u_{1}^{2}-v_{\sigma}^{2}}{u_{1}^{2}-u_{2}^{2}}\left(\frac{1}{\omega+u_{1} q}+\frac{1}{\omega-u_{1} q}\right)\right. \\
& \left.-\frac{u_{2}^{2}-v_{\sigma}^{2}}{u_{1}^{2}-u_{2}^{2}}\left(\frac{1}{\omega+u_{2} q}+\frac{1}{\omega-u_{2} q}\right)\right],
\end{aligned}
$$

which can be transformed into real space

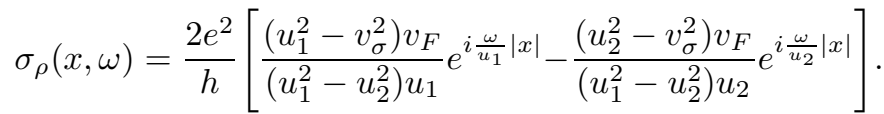

For convenience we use the abbreviation $\xi=x / l$, in which $\xi$ provides a dimensionless measured position in the wire and $l$ is the unit of length. Hence, Eq. (28) is reduced to

$\sigma_{\rho}(x, \omega)=\frac{2 e^{2}}{h}\left[\frac{\left(u_{1}^{2}-v_{\sigma}^{2}\right) v_{F}}{\left(u_{1}^{2}-u_{2}^{2}\right) u_{1}} e^{i \frac{\omega l}{u_{1}}|\xi|}-\frac{\left(u_{2}^{2}-v_{\sigma}^{2}\right) v_{F}}{\left(u_{1}^{2}-u_{2}^{2}\right) u_{2}} e^{i \frac{\omega l}{u_{2}}|\xi|}\right]$.

This result implicits that the ac charge conductivity of a perfect LL quantum wire with Rashba SOC is an oscillation function of the interaction parameter, SOC strength and the driving frequency as well as the measurement position in the wire. And in the limit of vanishing spinorbit and Zeeman coupling, the dc charge conductivity $\sigma_{\rho}=2 g e^{2} / h$ which is agreement with the conductivity of the previous studies ${ }^{18}$.

In addition, in the case of LL wire with Rashba SOC only, Eq. (22) for spin current operator is reduced to

$$
j_{\sigma}(q, \omega)=\frac{i e^{2} v_{F}}{\hbar \pi} \frac{2 \omega\left(-\delta v_{R} q \omega\right) E(q, \omega)}{\left(\omega^{2}-u_{1}^{2} q^{2}\right)\left(\omega^{2}-u_{2}^{2} q^{2}\right)},
$$

and similar calculation leads to the nonlocal spin conductivity for the system

$$
\sigma_{\sigma}(x, \omega)=\frac{2 e^{2}}{h} \frac{\delta v_{R} v_{F}}{u_{1}^{2}-u_{2}^{2}} \operatorname{sign}(\xi)\left(e^{i \frac{\omega l}{u_{1}}|\xi|}-e^{i \frac{\omega l}{u_{2}}|\xi|}\right),
$$

where $\operatorname{sign}(\xi)=-1$ for $\xi<0$ and 1 for $\xi>0$. This expression for spin conductivity has a less complicated dependence on the system parameters than charge conductivity (29). However, the spin conductivity vanishes as $\delta v_{R}=0$ or $\omega=0$.

Furthermore, if we reverse the transformation (4) and define the total (charge) conductivity $\sigma_{\rho}=\sigma_{\uparrow}+\sigma_{\downarrow}$ and the difference (spin) conductivity $\sigma_{\sigma}=\sigma_{\uparrow}-\sigma_{\downarrow}$, then the combination of Eqs. (29) and (31) gives

$$
\begin{aligned}
\sigma_{\uparrow} & =\frac{e^{2}}{h}\left[\left(\frac{\left(u_{1}^{2}-v_{\sigma}^{2}\right) v_{F}}{\left(u_{1}^{2}-u_{2}^{2}\right) u_{1}}+\frac{\delta v_{R} v_{F}}{u_{1}^{2}-u_{2}^{2}} \operatorname{sign}(\xi)\right) e^{i \frac{\omega l}{u_{1}}|\xi|}-\left(\frac{\left(u_{2}^{2}-v_{\sigma}^{2}\right) v_{F}}{\left(u_{1}^{2}-u_{2}^{2}\right) u_{2}}+\frac{\delta v_{R} v_{F}}{u_{1}^{2}-u_{2}^{2}} \operatorname{sign}(\xi)\right) e^{i \frac{\omega l}{u_{2}}|\xi|}\right] \\
& \stackrel{\omega \rightarrow 0}{\underline{h}} \frac{e^{2}}{h} \frac{v_{F}}{u_{1}^{2}-u_{2}^{2}}\left(\frac{u_{1}^{2}-v_{\sigma}^{2}}{u_{1}}-\frac{u_{2}^{2}-v_{\sigma}^{2}}{u_{2}}\right)
\end{aligned}
$$

and

$$
\begin{aligned}
\sigma_{\downarrow} & =\frac{e^{2}}{h}\left[\left(\frac{\left(u_{1}^{2}-v_{\sigma}^{2}\right) v_{F}}{\left(u_{1}^{2}-u_{2}^{2}\right) u_{1}}-\frac{\delta v_{R} v_{F}}{u_{1}^{2}-u_{2}^{2}} \operatorname{sign}(\xi)\right) e^{i \frac{\omega l}{u_{1}}|\xi|}-\left(\frac{\left(u_{2}^{2}-v_{\sigma}^{2}\right) v_{F}}{\left(u_{1}^{2}-u_{2}^{2}\right) u_{2}}-\frac{\delta v_{R} v_{F}}{u_{1}^{2}-u_{2}^{2}} \operatorname{sign}(\xi)\right) e^{i \frac{\omega l}{u_{2}}|\xi|}\right] \\
\stackrel{\omega \rightarrow 0}{\underline{ }} & \frac{e^{2}}{h} \frac{v_{F}}{u_{1}^{2}-u_{2}^{2}}\left(\frac{u_{1}^{2}-v_{\sigma}^{2}}{u_{1}}-\frac{u_{2}^{2}-v_{\sigma}^{2}}{u_{2}}\right)
\end{aligned}
$$

for the conductivity of spin-up and spin-down electrons, respectively. From Eqs. (32) and (33), we can see that in the case of $\omega=0$ or without Rashba SOC $\left(\delta v_{R}=0\right)$, the conductivities for the two spin subband are degenerate. Defining $v_{F \uparrow}=v_{F}-\delta v_{R} / 2\left(v_{F \downarrow}=v_{F}+\delta v_{R} / 2\right)$ as the Fermi velocity of the spin-up(down) subband in the presence of Rashba SOC, we can express $\delta v_{R} / v_{F}$ as

$$
\frac{\delta v_{R}}{v_{F}}=\frac{2\left(\frac{v_{F \downarrow}}{v_{F \uparrow}}-1\right)}{\frac{v_{F \downarrow}}{v_{F \uparrow}}+1},
$$

in which $v_{F \uparrow}=v_{F \downarrow}$ when $\delta v_{R}=0$.

\section{B. The conductivity with Zeeman splitting}

In this subsection we consider the case of the system with Zeeman splitting in the absence of SOC, i.e., in the case of $\alpha=0$ or $\delta v_{R}=0$. In this case the charge current operator reads

$$
j_{\rho}(q, \omega)=\frac{i e^{2} v_{F}}{\hbar \pi} \frac{2 \omega\left(\omega^{2}-v_{\sigma}^{2} q^{2}\right) E(q, \omega)}{\left[1-\left(\frac{\delta v_{B}}{2 v_{F}}\right)^{2}\right]\left(\omega^{2}-u_{1}^{2} q^{2}\right)\left(\omega^{2}-u_{2}^{2} q^{2}\right)},
$$


where

From this expression we can see that the ac conductivity of a perfect LL with only Zeeman splitting is also an os-

$u_{1,2}^{2}=\frac{v_{\rho}^{2}+v_{\sigma}^{2}+\frac{\delta v_{B}^{2}}{2}}{2\left[1-\left(\frac{\delta v_{B}}{2 v_{F}}\right)^{2}\right]} \pm \sqrt{\left(\frac{v_{\rho}^{2}+v_{\sigma}^{2}+\frac{\delta v_{B}^{2}}{2}}{2\left[1-\left(\frac{\delta v_{B}}{2 v_{F}}\right)^{2}\right]}\right)^{2}-\frac{v_{\rho}^{2} v_{\sigma}^{2}-\frac{\delta v_{B}^{2} v_{F}^{2}}{4} \text { cillation function of the interaction parameter, magnetic }}{1-\left(\frac{\delta v_{B}}{2 v_{F}}\right)^{2}} \text { field intensity and the driving frequency as well as the }}$

are the propagation velocities of collective modes. Again, when $\delta v_{B}=0$, they are also reduced to the velocities for the spin-charge separated system $u_{1,2}=v_{\rho, \sigma}{ }^{16,19}$

Additionally, through the same procedures as above, we obtain the result for the nonlocal charge conductivity

$$
\begin{aligned}
\sigma_{\rho}(q, \omega) & =\frac{i e^{2} v_{F}}{\hbar \pi} \frac{1}{1-\left(\frac{\delta v_{B}}{2 v_{F}}\right)^{2}}\left[\frac{u_{1}^{2}-v_{\sigma}^{2}}{u_{1}^{2}-u_{2}^{2}}\left(\frac{1}{\omega+u_{1}}+\frac{1}{\omega-u_{1}}\right)\right. \\
& -\frac{u_{2}^{2}-v_{\sigma}^{2}}{u_{1}^{2}-u_{2}^{2}}\left(\frac{1}{\omega+u_{2}}+\frac{1}{\omega-u_{2}}\right),
\end{aligned}
$$

Accordingly, using the same method above the spin conductivity for the LL wire only with Zeeman splitting is obtained as

$$
\begin{aligned}
\sigma_{\sigma}(x, \omega) & =\frac{2 e^{2}}{h} \frac{\frac{\delta v_{B}}{2 v_{F}}}{1-\left(\frac{\delta v_{B}}{2 v_{F}}\right)^{2}}\left[\frac{\left(u_{1}^{2}+v_{F}^{2}\right) v_{F}}{\left(u_{1}^{2}-u_{2}^{2}\right) u_{1}} e^{i \frac{\omega l}{u_{1}}|\xi|}\right. \\
& \left.-\frac{\left(u_{2}^{2}+v_{F}^{2}\right) v_{F}}{\left(u_{1}^{2}-u_{2}^{2}\right) u_{2}} e^{i \frac{\omega l}{u_{2}}|\xi|}\right] .
\end{aligned}
$$

which can be transformed into real space

$$
\begin{aligned}
\sigma_{\rho}(x, \omega) & =\frac{2 e^{2}}{h} \frac{1}{1-\left(\frac{\delta v_{B}}{2 v_{F}}\right)^{2}}\left[\frac{\left(u_{1}^{2}-v_{\sigma}^{2}\right) v_{F}}{\left(u_{1}^{2}-u_{2}^{2}\right) u_{1}} e^{i \frac{\omega l}{u_{1}}|\xi|}\right. \\
& \left.-\frac{\left(u_{2}^{2}-v_{\sigma}^{2}\right) v_{F}}{\left(u_{1}^{2}-u_{2}^{2}\right) u_{2}} e^{i \frac{\omega l}{u_{2}}|\xi|}\right] .
\end{aligned}
$$

We also see that as $\delta v_{B}=0$ the spin conductivity vanishes. Under the same definition of the total (charge) conductivity $\sigma_{\rho}=\sigma_{\uparrow}+\sigma_{\downarrow}$ and the difference (spin) conductivity $\sigma_{\sigma}=\sigma_{\uparrow}-\sigma_{\downarrow}$ with the combination of Eqs. (38) and (39), we can obtain

$$
\begin{gathered}
\sigma_{\uparrow}=\frac{e^{2}}{h} \frac{1}{1-\left(\frac{\delta v_{B}}{2 v_{F}}\right)^{2}} \frac{v_{F}}{u_{1}^{2}-u_{2}^{2}}\left[\left(\frac{u_{1}^{2}-v_{\sigma}^{2}}{u_{1}}+\frac{\delta v_{B}}{2 v_{F}} \frac{u_{1}^{2}+v_{F}^{2}}{u_{1}}\right) e^{i \frac{\omega l}{u_{1}}|\xi|}-\left(\frac{u_{2}^{2}-v_{\sigma}^{2}}{u_{2}}+\frac{\delta v_{B}}{2 v_{F}} \frac{u_{2}^{2}+v_{F}^{2}}{u_{2}}\right) e^{i \frac{\omega l}{u_{2}}|\xi|}\right] \\
\stackrel{\omega \rightarrow 0}{=} \frac{e^{2}}{h} \frac{1}{1-\left(\frac{\delta v_{B}}{2 v_{F}}\right)^{2}} \frac{v_{F}}{u_{1}^{2}-u_{2}^{2}}\left[\left(\frac{u_{1}^{2}-v_{\sigma}^{2}}{u_{1}}+\frac{\delta v_{B}}{2 v_{F}} \frac{u_{1}^{2}+v_{F}^{2}}{u_{1}}\right)-\left(\frac{u_{2}^{2}-v_{\sigma}^{2}}{u_{2}}+\frac{\delta v_{B}}{2 v_{F}} \frac{u_{2}^{2}+v_{F}^{2}}{u_{2}}\right)\right]
\end{gathered}
$$

and

$$
\begin{gathered}
\sigma_{\downarrow}=\frac{e^{2}}{h} \frac{1}{1-\left(\frac{\delta v_{B}}{2 v_{F}}\right)^{2}} \frac{v_{F}}{u_{1}^{2}-u_{2}^{2}}\left[\left(\frac{u_{1}^{2}-v_{\sigma}^{2}}{u_{1}}-\frac{\delta v_{B}}{2 v_{F}} \frac{u_{1}^{2}+v_{F}^{2}}{u_{1}}\right) e^{i \frac{\omega l}{u_{1}}|\xi|}-\left(\frac{u_{2}^{2}-v_{\sigma}^{2}}{u_{2}}-\frac{\delta v_{B}}{2 v_{F}} \frac{u_{2}^{2}+v_{F}^{2}}{u_{2}}\right) e^{i \frac{\omega l}{u_{2}}|\xi|}\right] \\
\stackrel{\underline{\underline{\omega \rightarrow 0}}}{\frac{e^{2}}{h}} \frac{1}{1-\left(\frac{\delta v_{B}}{2 v_{F}}\right)^{2}} \frac{v_{F}}{u_{1}^{2}-u_{2}^{2}}\left[\left(\frac{u_{1}^{2}-v_{\sigma}^{2}}{u_{1}}-\frac{\delta v_{B}}{2 v_{F}} \frac{u_{1}^{2}+v_{F}^{2}}{u_{1}}\right)-\left(\frac{u_{2}^{2}-v_{\sigma}^{2}}{u_{2}}-\frac{\delta v_{B}}{2 v_{F}} \frac{u_{2}^{2}+v_{F}^{2}}{u_{2}}\right)\right]
\end{gathered}
$$

for the conductivities of spin-up and spin-down electrons, respectively. We also see that without Zeeman splitting $\left(\delta v_{B}=0\right)$ the conductivities for the two spin subband are degenerate. Again, defining $v_{F \uparrow}=v_{F}+\delta v_{B} / 2\left(v_{F \downarrow}=\right.$ $v_{F}-\delta v_{B} / 2$ ) as the Fermi velocity of the up (down) spin subband in a magnetic field $B$, we can also express $\delta v_{B} / v_{F}$ as

$$
\frac{\delta v_{B}}{v_{F}}=\frac{2\left(\frac{v_{F \uparrow}}{v_{F \downarrow}}-1\right)}{\frac{v_{F \uparrow}}{v_{F \downarrow}}+1}
$$

which has the similar form as Eq. (34).

\section{RESULTS AND DISCUSSIONS}

There are six calculated figures presented in this paper, in which Fig. 1 to Fig. 3 are plotted for the system with Rashba SOC in the absence of an external magnetic field, whereas Fig. 4 to Fig. 6 are plotted for the system with Zeeman splitting in the absence of Rashba SOC.

The dimensionless velocities of the bosonic excitation $u_{1}\left(u_{2}\right)$ (in units of $v_{F}$ ) as a function of $\delta v_{R} / v_{F}$ calculated according to Eq. (25) in the absence of Zeeman splitting for three different electron-electron interaction strengths of $g=0.2$ (dotted line), 0.4 (dashed line) and 1 (solid line), respectively, are shown in Fig. 1. We can 

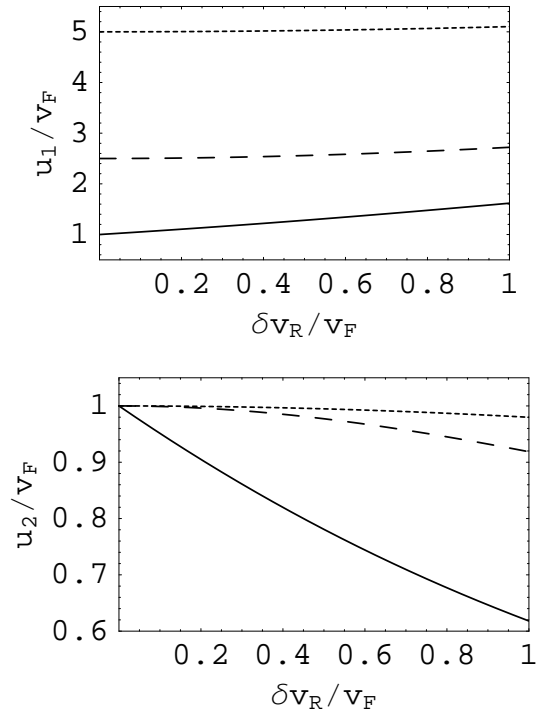

FIG. 1: The plotted propagation velocities of the collective modes $u_{1,2}$ (in units of $v_{F}$ ) as a function of the SOC strength $\delta v_{R} / v_{F}$ in the absence of Zeeman effect, where the solid lines for $g=1$, the dashed lines for $g=0.4$ and the dotted lines for $g=0.2$, respectively.

see that when the interaction is turned on $(g<1)$ and as $\delta v_{R} / v_{F}$ (proportional to Rashba SOC strength) increases $u_{1} / v_{F}$ increases while $u_{2} / v_{F}$ decreases slightly. However, for the stronger interaction the changes of $u_{1} / v_{F}$ and $u_{2} / v_{F}$ seem less obvious. For a fixed value of $\delta v_{R} / v_{F}$, the stronger the interaction is, the larger the velocities of the bosonic excitation $u_{1}\left(u_{2}\right)$ are. And in the limited case of $\delta v_{R} / v_{F}=0$ (i.e., in the absence of SOC), $u_{1} / v_{F}$ is equal to $1 / g$ whereas $u_{2} / v_{F}$ is equal to 1 . This is the known result that can be found in Ref. 20 .

Figure 2 illustrates the real part of charge conductivity $\operatorname{Re}_{\rho}(x, \omega)$ (in units of $e^{2} / h$ ) as a function of $\omega l / v_{F}$ calculated according to Eq. (29) in the absence of Zeeman splitting. For the system with fixed $g=0.25$ and $\delta v_{R} / v_{F}=0.5$, Fig. 2(a) shows the dependence of three different measurement positions $\xi= \pm 0.4$ (solid line), \pm 0.25 (dashed line) and 0 (dotted line) on the conductivity, respectively. In the center of the wire $(\xi=0)$ the conductivity is a constant value regardless of $\omega l / v_{F}$. However, the further off the wire center the position, the quicker the change of the conductivity is. Notice that the conductivity only depends on the absolute value $|\xi|$. The influence of the electron-electron interaction $g$ and the Rashba strength $\delta v_{R} / v_{F}$ on $\operatorname{Re} \sigma_{\rho}(x, \omega)$ for the system are shown in Fig. 2(b) and Fig. 2(c), respectively. For the system with fixed $\xi=0.25$ and $\delta v_{R} / v_{F}=0.5$, Fig. 2 (b) shows the dependence of three different interaction strengths $g=1$ (solid line), 0.75 (dashed line) and 0.25 (dotted line) on the conductivity, respectively. When the electron-electron interaction parameter $g$ is larger, the variation of $\operatorname{Re}_{\rho}(x, \omega)$ is faster. If the compositive vibration has a periodicity, then the stronger the inter-
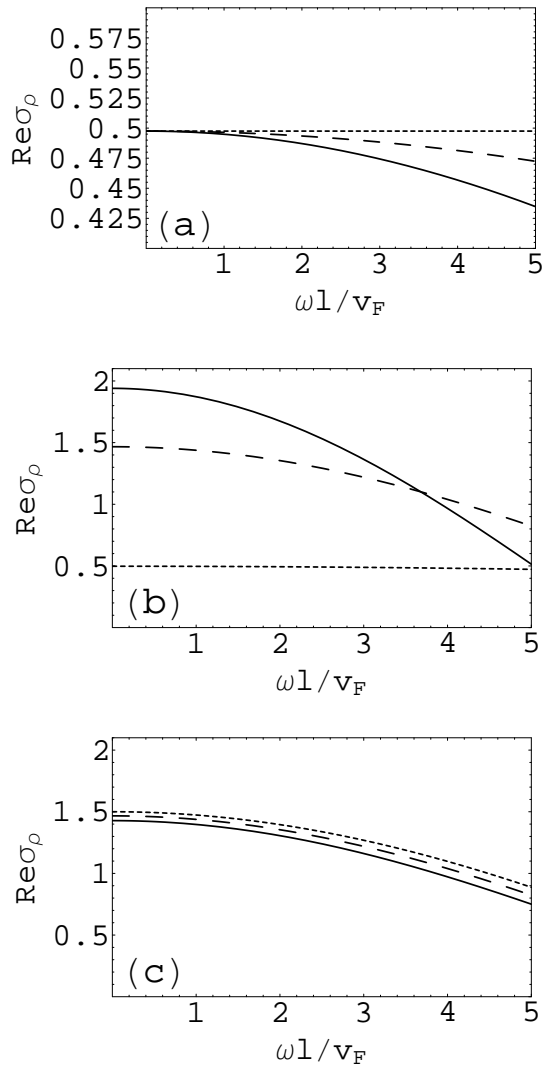

FIG. 2: The plotted $\operatorname{Re}_{\rho}(x, \omega)$ (in units of $e^{2} / h$ ) as a function of $\omega l / v_{F}$ in the absence of Zeeman effect (a) with fixed $g=$ 0.25 and $\delta v_{R} / v_{F}=0.5$ where the solid line for $\xi= \pm 0.4$, the dashed line for $\xi= \pm 0.25$ and the dotted line for $\xi=0$; (b) with fixed $\xi=0.25$ and $\delta v_{R} / v_{F}=0.5$ where the solid line for $g=1$, the dashed line for $g=0.75$ and the dotted line for $g=0.25$; and (c) with fixed $\xi=0.25$ and $g=0.75$ where the solid line for $\delta v_{R} / v_{F}=0.75$, the dashed line for $\delta v_{R} / v_{F}=0.5$ and the dotted line for $\delta v_{R} / v_{F}=0$, respectively.

action is, the longer the period of the oscillation is. The period of the oscillation is the least common multiple of $2 \pi u_{1} /\left(v_{F}|\xi|\right)$ and $2 \pi u_{2} /\left(v_{F}|\xi|\right)$, and a measured position is fixed $\xi=0.25$, so the period of the oscillation is totally determined by $u_{1}$ and $u_{2}$. For the system with fixed $g=0.75$ and $\xi=0.25$, Fig. 2(c) shows the dependence of three different Rashba strengths $\delta v_{R} / v_{F}=0.75$ (solid line), 0.5 (dashed line) and 0 (dotted line) on the conductivity, respectively. Comparing Fig. 2(c) with 2(b), we can find that the dependence of $\operatorname{Re}_{\rho}(x, \omega)$ on $g$ at fixed $\delta v_{R} / v_{F}$ is very similar to that on $\delta v_{R} / v_{F}$ at fixed $g$, and they exhibit the same tendency as a function of $\omega l / v_{F}$. But it is obvious that the modification due to the electron-electron interactions is remarkable. Moreover, from Figs. 2(b) and 2(c), we can see that the dc $(\omega=0)$ charge conductivities of the system with different electron-electron interactions and Rashba strengths are different constant values, which can be obtained analyt- 


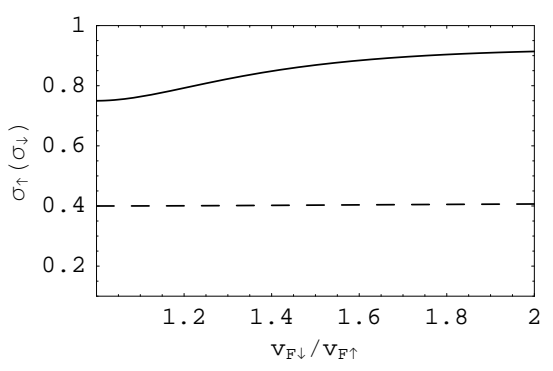

FIG. 3: The plotted spin-polarized charge conductivity $\sigma_{\uparrow}\left(\sigma_{\downarrow}\right)$ (in units of $e^{2} / h$ ) as a function of the ratio $v_{F \downarrow} / v_{F \uparrow}$ in the absence of Zeeman effect, where the solid lines correspond to $g=0.75$ and the dashed lines to $g=0.4$, respectively.

ically from Eq.(29) with $\lim _{\omega \rightarrow 0} \sigma_{\rho}(x, \omega)=2 e^{2} / h\left[\left(u_{1}^{2}-\right.\right.$ $\left.\left.v_{\sigma}^{2}\right) v_{F} / u_{1} /\left(u_{1}^{2}-u_{2}^{2}\right)-\left(u_{2}^{2}-v_{\sigma}^{2}\right) v_{F} / u_{2} /\left(u_{1}^{2}-u_{2}^{2}\right)\right]$. Here $u_{1}$ and $u_{2}$ are dependent on both $g$ and $\alpha$ (see Fig. 1).

In Fig. 3 we show the dependence of spin-polarized dc conductivity $\sigma_{\uparrow}\left(\sigma_{\downarrow}\right)$ on the ratio $v_{F \downarrow} / v_{F \uparrow}$ (also proportional to Rashba SOC strength) in the absence of Zeeman splitting for the two different interaction strengths, where the solid lines correspond to $g=0.75$ and the dashed lines to $g=0.4$, respectively. The curves for $\sigma_{\downarrow}$ as the function of $v_{F \downarrow} / v_{F \uparrow}$ are the same as those for $\sigma_{\uparrow}$, and this can be verified from Eqs. (32) and (33). This result implies that the dc conductivities of spin-up and spin-down electrons are degenerate in the absence of Zeeman effect. From Fig. 3 we can also see that the spin-polarized conductivities $\sigma_{\uparrow}\left(\sigma_{\downarrow}\right)$ is connected with the electron-electron interactions for any fixed value of $v_{F \downarrow} / v_{F \uparrow}$. The stronger the interactions produce the smaller $\sigma_{\uparrow}\left(\sigma_{\downarrow}\right)$ which show that the repulsive interaction suppresses the conductivity. And the less obvious the increase of $\sigma_{\uparrow}\left(\sigma_{\downarrow}\right)$ as the increases of ratio $v_{F \downarrow} / v_{F \uparrow}$, which show that in the case of strong electron-electron interaction the modification of $v_{F \downarrow} / v_{F \uparrow}$ on the conductivity is very little.

Figure 4 shows the dimensionless velocities of the bosonic excitations $u_{1}\left(u_{2}\right) / v_{F}$ vs $\delta v_{B} / v_{F}$ of the system calculated according to Eq. (36) in the absence of Rashba SOC for three different interaction strengths of $g=1$ (solid line), 0.4 (dashed line) and 0.2 (dotted line). We also see that with the increase of $\delta v_{B} / v_{F} u_{1} / v_{F}$ increases while $u_{2} / v_{F}$ decreases slightly for all values of $g<1$. But for noninteracting case $(g=1) u_{2} / v_{F}$ decays more rapidly as $\delta v_{B} / v_{F}$ increases, and the stronger the interaction is, the slower the decay of $u_{2} / v_{F}$. Fig. 4 is very similar to Fig. 1, which makes clear that the virtual magnetic field induced by an electric field perpendicular to the 2DEG yields the similar effect on the propagation velocities of the collective modes as the magnetic field applied along the $y$ direction.

Figure 5 illustrates the real part of charge conductivity $\operatorname{Re}_{\rho}(x, \omega)$ (in units of $e^{2} / h$ ) as a function of $\omega l / v_{F}$ calculated according to Eq. (38) in the absence of Rashba SOC. For the system with fixed $g=0.25$ and $\delta v_{B} / v_{F}=$ 0.5 , Fig. 5(a) shows the dependence of three different measurement positions $\xi= \pm 0.4$ (solid line), \pm 0.25
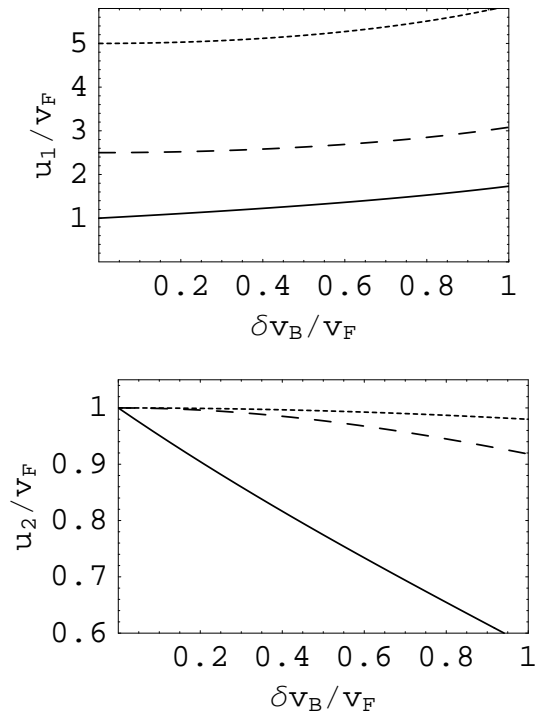

FIG. 4: The plotted propagation velocities of the collective modes $u_{1,2}$ (in units of $v_{F}$ ) as a function of the Zeeman strength $\delta v_{B} / v_{F}$ in the absence of SOC, where the solid lines correspond to $g=1$, the dashed lines to $g=0.4$ and the dotted lines to $g=0.2$, respectively.

(dashed line) and 0 (dotted line) on the conductivity, respectively. Figure 5(b) shows the dependence of three different interaction strengths $g=1$ (solid line), 0.75 (dashed line) and 0.25 (dotted line) on the conductivity for the system with fixed $\xi=0.25$ and $\delta v_{B} / v_{F}=0.5$. And for the system with fixed $g=0.75$ and $\xi=0.25$, Fig. 5(c) shows the dependence of three different magnetic strengths $\delta v_{B} / v_{F}=0.75$ (solid line), 0.5 (dashed line) and 0 (dotted line) on the conductivity, respectively. The variables and the scales in Fig. 5 are the same as that in Fig. 2 except replacing $\delta v_{R} / v_{F}$ by $\delta v_{B} / v_{F}$. And Fig. 5 is very similar to Fig. 2. However, from Fig. 5 we can see that the dc $(\omega=0)$ charge conductivities of the system with different electron-electron interactions or magnetic strengths are different constant values [Fig. 5(b) and 5(c)], but with different measurement positions are the same values [Fig. 5(a)]. This is because that $\lim _{\omega \rightarrow 0} \sigma_{\rho}(x, \omega)=2 e^{2} / h\left(1 /\left[1-\left(\delta v_{B} /\left(2 v_{F}\right)\right)^{2}\right]\right)\left[\left(u_{1}^{2}-\right.\right.$ $\left.\left.v_{\sigma}^{2}\right) v_{F} / u_{1} /\left(u_{1}^{2}-u_{2}^{2}\right)-\left(u_{2}^{2}-v_{\sigma}^{2}\right) v_{F} / u_{2} /\left(u_{1}^{2}-u_{2}^{2}\right)\right]$ where $u_{1}$ and $u_{2}$ are only dependent on both $g$ and $\delta v_{B}$ (see Fig. 4). These constant values are almost the product of $\operatorname{Re} \sigma_{\rho}(x, \omega=0)$ in Fig. 2 and a factor $1 /\left[1-\left(\delta v_{B} / 2 v_{F}\right)^{2}\right]$, and is also true in the case of $\omega \neq 0$. Comparing Fig. 5(c) with 5(b), we can find that the dependence of $\operatorname{Re}_{\rho}(x, \omega)$ on $g$ at fixed $\delta v_{B} / v_{F}$ is very similar to that on $\delta v_{B} / v_{F}$ at fixed $g$, and they exhibit the same tendency as a function of $\omega l / v_{F}$. But it is obvious that the modification due to the electron-electron interactions is more remarkable, which is the same as the conclusion by comparing Fig. 2(c) with 2(b). Moreover comparing Fig. 5(c) with $2(\mathrm{c})$, we can find that the dependence of $\operatorname{Re}_{\rho}(x, \omega)$ on $\delta v_{B} / v_{F}$ is very similar to that on $\delta v_{R} / v_{F}$ in the case of 

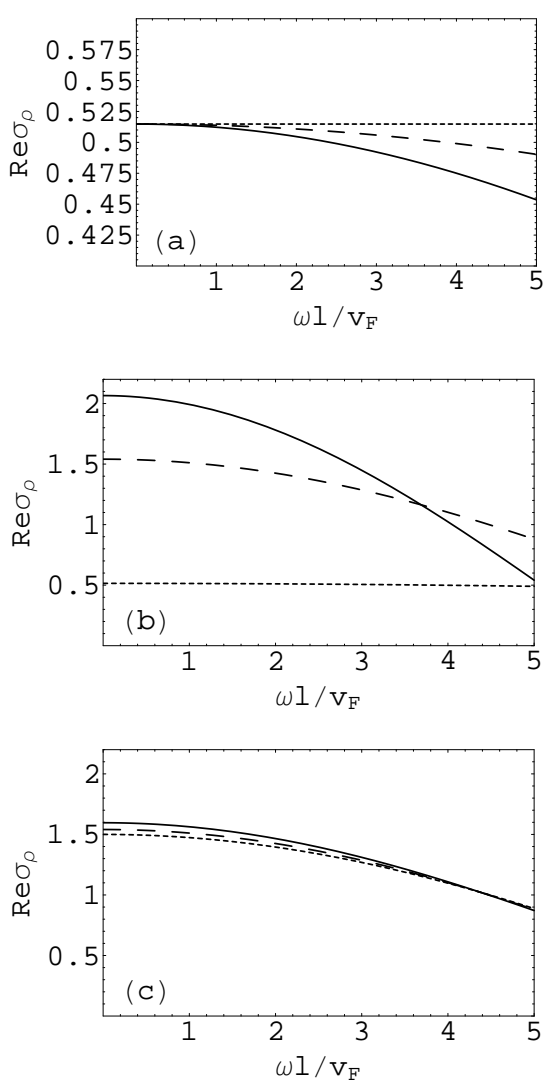

FIG. 5: The plotted $\operatorname{Re}_{\rho}(x, \omega)$ (in units of $e^{2} / h$ ) as a function of $\omega l / v_{F}$ in the absence of SOC (a) with fixed $g=0.25$ and $\delta v_{B} / v_{F}=0.5$ where the solid line for $\xi= \pm 0.4$, the dashed line for $\xi= \pm 0.25$ and the dotted line for $\xi=0$; (b) with fixed $\xi=0.25$ and $\delta v_{B} / v_{F}=0.5$ where the solid line for $g=1$, the dashed line for $g=0.75$ and the dotted line for $g=0.25$; and (c) with fixed $\xi=0.25$ and $g=0.75$ where the solid line for $\delta v_{B} / v_{F}=0.75$, the dashed line for $\delta v_{B} / v_{F}=0.5$ and the dotted line for $\delta v_{B} / v_{F}=0$, respectively.

fixed $g$, and they exhibit the same tendency as a function of $\omega l / v_{F}$. This means that the effects of Rashba SOC and Zeeman splitting on the conductivity are very similar.

In Fig. 6 we show how the spin polarized dc conductivities $\sigma_{\uparrow}$ and $\sigma_{\downarrow}$ evolve as the ratio of $v_{F \uparrow} / v_{F \downarrow}$ is varied for the two different interaction strengths, where the solid lines correspond to $g=0.75$ and the dashed lines to $g=0.4$, respectively. It is also demonstrated that the increase of ratio $v_{F \uparrow} / v_{F \downarrow}$ pushes $\sigma_{\uparrow}$ and $\sigma_{\downarrow}$ away from each other, and one accelerates while the other slows down. This result is in agreement with Ref. 9. In contrast to the Rashba SOC case as shown in Fig. 3, the ratio of the spin-polarized conductivities $\sigma_{\uparrow} / \sigma_{\downarrow}$ is dependent on the electron-electron interactions and the ratio $v_{F \uparrow} / v_{F \downarrow}$. This is because the channel with a larger electron velocity has a larger transmission coefficient for the fixed electron-electron interaction strength and $v_{F \downarrow} / v_{F \uparrow}$, and the difference of the transmission coefficient between

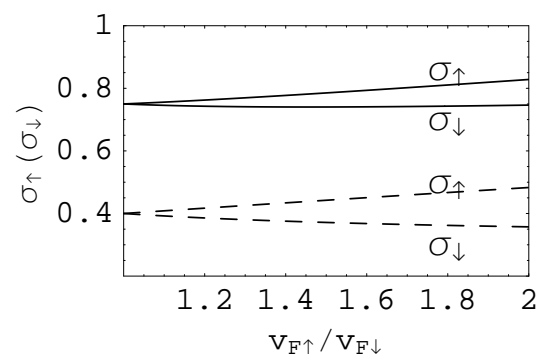

FIG. 6: The plotted spin-polarized charge conductivity $\sigma_{\uparrow}\left(\sigma_{\downarrow}\right)$ (in units of $e^{2} / h$ ) as a function of the ratio $v_{F \uparrow} / v_{F \downarrow}$ in the absence of SOC, where the solid lines correspond to $g=0.75$ and the dashed lines to $g=0.4$, respectively.

channels becomes larger with the increase of $v_{F \downarrow} / v_{F \uparrow}$ or of electron-electron interaction strength.

Finally, in II-VI semiconductors the Rashba SOC is expected to be larger than the Dresselhaus coupling, so we can neglect the Dresselhaus SOC. ${ }^{8}$ At low temperatures, 2DEG formed in II-VI semiconductor heterostructures is restricted by a transverse confining potential, so we have the narrow long enough quantum wire. A weak magnetic field $B$ perpendicular to the quantum wire is applied along $y$-axis, which is turned on or turned off according to the requirement. A longitudinally polarized external electromagnetic field with wavevector along the $z$ axis irradiates the quantum wire. For the aforementioned conditions, the present experiment can be available. ${ }^{2-4}$ Furthermore, in this article we have only considered the case of infinite-length LL. The situation is important for a simple theoretical understanding, although it may be relevant to experiments where the leads always dominate the results. The conductivity of a finite-size LL coupled to leads may have different frequency and amplitude dependence on the physical parameters of the system due to the Andreev-type reflections. ${ }^{19}$ The detailed calculation and discussion for the important role the Fermi liquid leads on the conductivity of the system will be given in our next work.

\section{CONCLUSION}

In conclusion, using a straightforward approach we have investigated theoretically the transport properties through an interacting quantum wire in the presence of both Rashba SOC and Zeeman splitting simultaneously in the LL regime. Using the bosonization technique, the motion of equations of bosonic phase fields for the system with a longitudinal electric field is established, and its solution is obtained by introducing a Fourier transformation in which the spin and charge degrees of freedom are completely coupled and characterized by four new different velocities. Within the linear response theory, it is found that the ac conductivity of a LL wire in the presence of Rashba SOC and Zeeman splitting is generally an 
oscillation function of the interaction strength $g$, Rashba SOC strength $\alpha$, Zeeman interaction strength $B$ and the driving frequency $\omega$ as well as the measurement position $x$ in the wire.

For a LL wire only with Rashba SOC, the real part of the conductivity $\operatorname{Re}_{\rho}$ as the function of the electronelectron interaction or Rashba SOC strength exhibits the similar decay tendencies with the increase of frequency $\omega l / v_{F}$. But the modification due to the electron-electron interactions is more remarkable than that due to Rashba SOC. On the other hand, for a LL wire only with Zeeman splitting. On the other hand, for a LL wire only with Zeeman splitting, $\operatorname{Re}_{\rho}$ is a function of the frequency $\omega l / v_{F}$, the same curves hold if one replaces $\delta v_{R} / v_{F}$ by $\delta v_{B} / v_{F}$, but one has to multiply all values of $R e \sigma_{\rho}$ by a factor of $1 /\left[1-\left(\delta v_{B} / 2 v_{F}\right)^{2}\right]$.

For a LL only with Rashba SOC, when we study how $\sigma_{\uparrow}\left(\sigma_{\downarrow}\right)$ evolve as the ratio $v_{F \downarrow} / v_{F \uparrow}$ is varied, we find that the curves for $\sigma_{\downarrow}$ as the function of $v_{F \downarrow} / v_{F \uparrow}$ are the same as those for $\sigma_{\uparrow}$. But for a LL only with Zeeman splitting, we find that the increase of the ratio $v_{F \uparrow} / v_{F \downarrow}$ pushes $\sigma_{\uparrow}$ and $\sigma_{\downarrow}$ away from each other, which is consistent with that of the previous studies ${ }^{9}$ for the same system. In contrast to the SOC case, the ratio of the spin-polarized conductivities $\sigma_{\uparrow} / \sigma_{\downarrow}$ is dependent on the electron-electron interactions.

Further investigations are worthy to be done for the higher conductivity corrections, in the presence of impurity, or with realistic Coulomb interactions.

\section{Acknowledgments}

This work was supported by National Natural Science Foundation of China (Grant No. 10574042), Specialized Research Fund for the Doctoral Program of Higher Education of China (Grant No. 20060542002) and Hunan Provincial Natural Science Foundation of China (Grant No. 06JJ2097).
* Electronic address: ghzhou@hunnu.edu.cn

1 Voit J 1995 Rep. Prog. Phys. 58977

2 Yacoby A, Stormer H L, Wingreen N S, Pfeiffer L N, Baldwin K W and West K W 1996 Phys. Rev. Lett. 774612

3 Bockrath M, Cobden D H, Lu J, Rinzler A G, Smalley R E, Balents L and McEuen P L 1999 Nature 397598

${ }^{4}$ Chang. A. M, Pfeiffer. L. N and K. W. West 1996 Phys. Rev. Lett. 772538

5 Wolf S A, Awschalom D D, Buhrman R A, Daughton J M, von Molnár S, Roukes M L, Chtchelkanova A Y and Treger D M 2001 Science 2941488

6 Bychkov Y A and Rashba E I 1984 J. Phys. C 176039

7 Dresselhaus G 1955 Phys. Rev. 100580

8 Nitta J, Akazaki T, Takayanagi H and Enoki T 1997 Phys. Rev. Lett. 781335

${ }^{9}$ Kimura T, Kuroki K and Aoki H 1996 Phys. Rev. B 53 9572

10 Moroz A V, Samokhin K V and Barnes C H W 2000 Phys. Rev. Lett. 84 4164; 2000 Phys. Rev. B 6216900

11 Iucci A 2003 Phys. Rev. B 68075107

12 Gritsev V, Japaridze G, Pletyukhov M and Baeriswyl D 2005 Phys. Rev. Lett. 94137207

13 Hankiewicz E M and Vignale G 2006 Phys. Rev. B 73 115339
14 Yu Yue, Wen Yuchuan, Li Jinbi, Su Zhaobin and Chui S T 2004 Phys. Rev. B 69153307

15 Devillard P, Crepieux A, Imura K I and Martin T 2005 Phys. Rev. B 72 R041309

16 Ponomarenko V V 1996 Phys. Rev. B 54 10328; Sharma $\mathrm{P}$ and Chamon C 2003 ibid 68 035321; Feldman D E and Gefen Y 2003 ibid 67 115337; Cheng F and Zhou G H 2006 ibid $\mathbf{7 3} 125335$

17 Sassetti M and Kramer B 1996 Phys. Rev. B 54 R5203

18 Cuniberti G, Sassetti M and Kramer B 1998 Phys. Rev. B 57 1515; Fechner A, Sassetti M, Kramer B and Galleani d'Agliano E 2001 ibid 64195315

19 Maslov D L and Stone M 1995 Phys. Rev. B 52 R5539; Ponomarenko V V 1995 ibid 52 R8666; Maslov D L 1995 ibid 52 R14368; Safi I and Schulz H J 1995 ibid 52 R17040; Sandler N P, Chaman C C and Frakin E 1998 ibid 57 12324; Dolcini F, Trauzettel B, Safi I and Grabert H 2005 ibid 71 165309; Janzen K, Meden V and Schönhammer K 2006 ibid 74085301

20 Gogolin A O, Nersesyan A A and Tsvelik A M 1998 Bosonization and Strongly Correlated Systems (Cambridge University Press, Cambridge) 